\title{
Author Correction: Dynamic thylakoid stacking regulates the balance between linear and cyclic photosynthetic electron transfer
}

William H. J. Wood (D, Craig MacGregor-Chatwin, Samuel F. H. Barnett (D), Guy E. Mayneord, Xia Huang, Jamie K. Hobbs,

C. Neil Hunter (D) and Matthew P. Johnson (D)

Correction to: Nature Plants https://doi.org/10.1038/s41477-017-0092-7, published online 29 February 2018.

In the version of this Article originally published, the authors incorrectly labelled the timescale in Fig. 6b as milliseconds (ms) on the $x$ axis and the indicated half-life values; the correct units are microseconds ( $\mu \mathrm{s})$. The figure has now been amended in all versions of the Article. 